\title{
Enhancement of Retinex Algorithm using Fast Fourier Transform
}

\author{
Muazzamu Ibrahim \\ Computer vision and Robotics \\ Dept. \\ Changchun University of Science \\ and Technology
}

\author{
Zayyanu Shuaibu \\ Brain computing Dept. \\ Changchun University of Science \\ and Technology
}

\author{
Nura M. Bello \\ Computer vision and Robotics \\ Dept. \\ Changchun University of Science \\ and Technology
}

\begin{abstract}
Image processing plays an important role in various field of human endeavor. Retinex method is widely used to improve the quality of the processed images. It provides better dynamic range compression, lightness rendition and color constancy.it also aimed at mimicking human vision system and lead to efficient algorithm for enhancing local image contrast. Various researchers proposed distinctive methods for enhancing image quality. The oldest and most popular image enhancement algorithm is histogram equalization which is used to improve the contrast of image. Other methods are Single-Scale and Multi-Scale Retinex (MSR) used for image quality enhancement, but cause some halation problem, high computation time and does not preserve edges. Recursive bilateral filter can able to do its best for solving the problems faced in previous algorithms but still has high computation time. In this paper, we proposed fast and efficient method for improving the quality of an image and reducing the computational time using three main steps: 1. Apply Fast Fourier transform to the input image, to convert the image frequency domain from spatial domain and multiply gaussian filter with illumination in frequency domain, this will speed up the processing time and provide better result. 2. Retinex algorithm is used to estimate the reflectance by subtracting illumination image from original image. 3. Bi-histogram equalization to improve its visual effect. Lastly, the algorithm is evaluated from subjective and objective aspect. Experimental results demonstrate the effectiveness of the proposed method.
\end{abstract}

\section{Keywords}

Image enhancement, Retinex algorithm, Fast Fourier transform, Bi-histogram equalization

\section{INTRODUCTION}

Image enhancement is one of the most interested area in the field of computer vision domain for improving image quality. Image enhancement has been widely applicable in various field, especially in medical and transportation. For example, in medicine is used for diagnosis of patient, and in transportation for clearing path for self-driving car in bad weather conditions.

The oldest and most popular methods of image enhancement algorithms are histogram equalization, low-pass filtering and high-pass filtering which are applicable to some specific area hence, have some drawbacks [16]. Low-pass filtering and high-pass filtering can only sharpen and smoothen the image respectively [8]. Histogram equalization (HE) is used for image enhancement by distributing image intensity value uniformly and is good for preserving contrast and brightness of an image. Though has some limitations such as abnormal contrast enhancement and inadequate for local details resulting in partial details lost [9][1]. Bi-histogram equalization (BBHE) [2], dualistic sub-image histogram equalization (DSIHE) [3], and contrast limited adaptive histogram equalization (CLAHE) [10] methods has been proposed to improve the local details of image and to prevents over-enhancement by improving the low light image and speed up processing [10].

Retinex algorithm is a non-linear spectral that mimic the human vision system and provides simultaneous dynamic range compression and color constancy [11], The algorithm treats image as a product of illumination and reflectance. This theory module was first brought by Edwin Land in 1977, and thereafter Jobson.et extended two Algorithms; Single-Scale Retinex (SSR) and Multi-scale algorithm [4], which the final output is the reflectance component. SSR can either provide small dynamic range compression or wide tonal rendition [4]. MSR is an extension of SSR that provides both dynamic range compression and color rendition due to its combination of different weighted scales of SSR. The accurate the scale value, number and weight chosen, the better result of MSR algorithm [12]. Multi-Scale Retinex (MSR) algorithm is used to process each RGB color channel independently, the processed image would have unexpected color distortion. Then, a Multi-Scale Retinex Algorithm with Color Restoration (MSRCR) is proposed, introducing a color restoration factor to correct the color distortion [4-5]. Although MSRCR has achieved some effects in color rendition but does not solve the problem of color distortion completely because halation may appear in the high contrast area. In order to solve the halation, Bilateral filtering based on the Retinex algorithm is introduced, this solved the problem though takes long time to process image, therefore not suitable for real-time system [6]. A Retinex algorithm for image enhancement based on recursive bilateral filtering is proposed to solve the problems of previous algorithm, problems almost solved but still needs improvement especially in the computational time [8]. Notably, almost all the enhancement algorithms have their own cons and prons.

In this paper, we proposed fast and optimal way of image enhancement algorithm based on Retinex method for improving the quality such as sharpening, smoothing, reducing computational time and details of image by using Fast Fourier Transport with Retinex and Bi-histogram equalization.

\section{RETINEX THEORY}

Retinex is a combined word of Retina and Cortex, the theoretical definition of Retinex is to mimic human vision system. The model provides color constancy and tonal rendition. This theory was first developed by Edwin Land in 1977, and later Jobson.et al proposed two most popular 
Retinex algorithms called Single Scale Retinex (SSR) and Multiple Scale Retinex (MSR) [8][15]. In this model, the image $S(x, y)$ is the product of illumination and reflectance. The general model of Retinex algorithm is given by

$$
S(x, y)=R(x, y) * L(x, y)
$$

Where $\mathrm{S}_{(x, y)}$ is an input image and known, while $\mathrm{R}(x, y)$ is to be solved and $\mathrm{L}(x, y)$ is the incident light to be estimated using Gaussian filter.

\subsection{Single Scale Retinex}

Single Scale Retinex an image enhancement algorithm where output image is determined by the difference between the input image value and average of its neighborhood [14]. In Jobson et al.'s SSR, the illumination $l_{i}(x, y)$ is first estimated by applying a Gaussian-form linear LPF to an input color image $\mathrm{Si}(\mathrm{x}, \mathrm{y})$, the output color image $\mathrm{Ri}(\mathrm{x}, \mathrm{y})$ is then obtained by subtracting the log signal of the estimated illumination $\log \mathrm{l}_{\mathrm{i}}(\mathrm{x}, \mathrm{y})$ from the $\log$ signal of input color image $\log \mathrm{Si}(\mathrm{x}, \mathrm{y})[13]$.

SSR for an image $S(x, y)$ is defined as;

$$
\begin{gathered}
\operatorname{Ri}(x, y)=\log \operatorname{Si}(x, y)-\log [F(x, y) * \operatorname{Si}(x, y)], i \\
=1, \ldots S
\end{gathered}
$$

By using Gaussian surround function to $\mathrm{F}(\mathrm{x}, \mathrm{y})$, the normalized surround function,

$$
F(x, y)=K e^{-\left\{\frac{(X * X)+(Y * Y)}{C * C}\right\}}
$$

where $\mathrm{c}$ is the Gaussian surround constant, that is referred to as the scale of the SSR,

and $\mathrm{K}$ is selected such that

$$
\iint F(x, y) d x d y=1
$$

This algorithm can provide either dynamic range compression or color rendition

\subsection{Multi-Scale Retinex}

Multi-scale Retinex (MSR) algorithm is an extension of the single scale Retinex algorithm, proposed by Jobson et al. Due to the trade-off between dynamic range compression and color rendition, the choice of the right scale $\sigma$ for the surround filter $\mathrm{F}(\mathrm{x}, \mathrm{y})$ is crucial in SSR, MSR seems to afford an acceptable trade-off between a good local dynamic range and a good color rendition. The MSR output is defined as a weighted sum of the outputs of several SSRs. The multiscale Retinex formula can be represented as;

$R_{M S R i}=\sum_{n=1}^{N}(W n R n i)$

Where

$\operatorname{Ri}(\mathrm{x}, \mathrm{y})=\log \operatorname{Si}(x, y)-\log [F(x, y) * \operatorname{Ii}(x, y)], i=1, \ldots s, \mathrm{n}$ is the number of scales, Rni is the ith component of the nth scale, $\mathrm{R}_{\mathrm{MSRi}}$ is the ith spectral component of the MSR output, and $\mathrm{Wn}$ is the weight associated with the nth scale. The surround function is the same as that of SSR. And the algorithm can achieve both dynamic range compression and color rendition simultaneously but has some halation problem.

\section{PROPOSED SYSTEM}

\subsection{Fast Fourier Transform}

In the proposed system, the first step is to compute the Fast Fourier Transform (FFT) of the input image in spatial domain, the result of FFT is in frequency domain that does not contain all frequencies forming an image, but only a set of samples which is large enough to fully describe the spatial domain image. Once the image is transformed into the frequency domain, filters can be applied to the image by convolution. FFT turns the complicated convolution operations into simple multiplications. An inverse transform is then applied in the frequency domain to obtain the result of convolution. The resultant image contains all the important details. Applying filters to image in frequency domain is computationally faster than to do the same in the image domain. This will speed up the process and provide better result. These are the steps followed:

a) Read and resize input image.

b) Separate each color channels (RGB)

c) Computes FFT of gaussian filter.

d) Computes FFT of each color band RGB

e) Multiply each [d] with $(-1)^{\wedge x+y}$ in order to centralize the transform or use MATLAB function.

f) Multiply each [e] by [c]

g) Decentralize and computes the inverse of each [f] so that the image be in spatial domain rather frequency domain.

The Fast Fourier transport of image $\mathrm{S}(\mathrm{x}, \mathrm{y})$ is denoted by $\mathrm{F}(\mathrm{u}, \mathrm{v})$, is given by the equation:

$$
f(u, v)=\sum_{x=0}^{M-1} \sum_{y=0}^{N-1} S(x, y) e^{-j 2 \pi\left(\frac{u x}{M}+\frac{v y}{N}\right)}
$$

Where $\mathrm{x}=0,1,2 \ldots, \mathrm{M}-1$ and $\mathrm{y}=0,1,2 \ldots, \mathrm{N}-1$ denoted a digital image of size $M \times N$ pixels. And $u=0,1,2 \ldots, M-1$ and $\mathrm{v}=0,1,2 \ldots, \mathrm{N}-1$ which determine the frequencies of $\mathrm{x}$ and $\mathrm{y}$, $\mathrm{S}(\mathrm{x}, \mathrm{y})$ is the image in spatial domain and $\mathrm{F}(\mathrm{u}, \mathrm{v})$ the exponential term is the basis function corresponding to each point $F(u, v)$ in the Fourier space. The $F(u, v)$ is obtained by multiplying image in spatial domain with the corresponding based function and summing the result. This indicated that the number of frequencies in frequency domain is equal to the number of pixels in spatial domain that is, the image in spatial domain and frequency domain are of the same size. The result of FFT is a complex number which has large range than the image in spatial domain. Furthermore, the dynamic range of Fourier coefficients are too large to display on the screen, hence these values has to be scaled to bring them within the range of the values that can be displayed [7].

The FFT can be re-transform to a spatial domain by taking the inverse which is given by:

$$
S(x, y)=1 / M N \sum_{u=0}^{M-1} \sum_{v=0}^{N-1} F(u, v) e^{j 2 \pi\left(\frac{u x}{M}+v y / N\right)}
$$

Where $1 / \mathrm{MN}$ is the normalization term in the inverse transformation. This normalization is sometimes applied to the forwad transform instead of the inverse transform [14].

\subsubsection{Algorithm flow chart.}

The figure 1 below describe the step by step method for processing image in frequency domain using the proposed Fast Fourier Transform. 


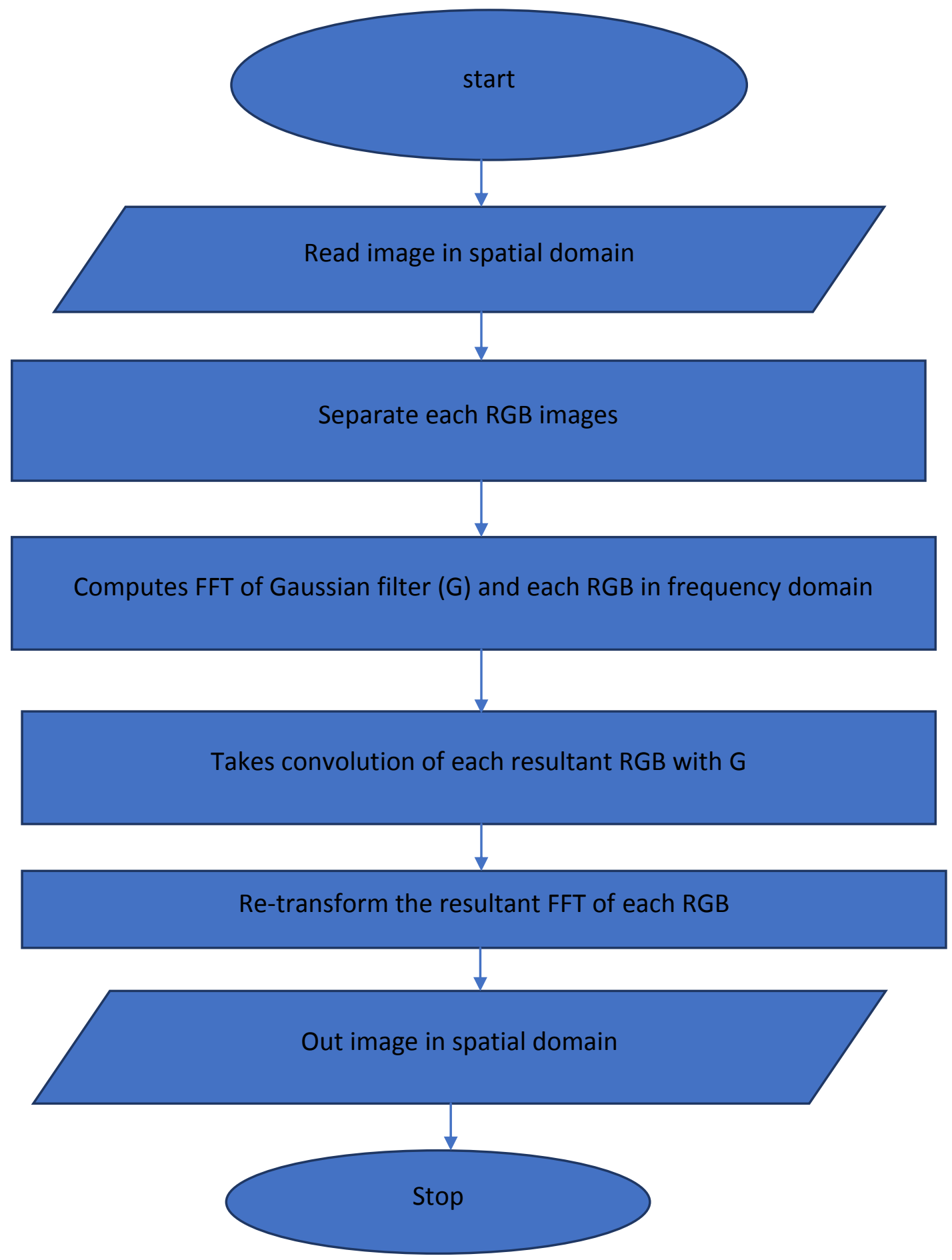

Figure 1 first part flow chart of the proposed system

Figure 1 is the first part of the main proposed system algorithm; it's started by reading the image. In case of color image, its treats each RGB independently than Fast Fourier Transform is applied to the image. This is the most interested part in the proposed algorithm that make it differ from existing algorithms. The gaussian filter is multiply with each RGB and the output is obtained by taking inverse of Fast Fourier Transform.

\subsection{Main Algorithm}

In the proposed algorithm, the input image has to undergo Fast Fourier Transform to regain some of the features lost. The Fourier Transform a complex number valued output image which can be displayed with two images, either with the real and imaginary part or with magnitude and phase. in this system, we focus on the displaying magnitude of the Fourier $\operatorname{Tr}$ produces ansform as it contains most of the information of the geometric structure of the spatial domain image. The resultant magnitude is multiplying with gaussian filter which smooth and sharpen the image. Furthermore, we re-transform the Fourier image into the correct spatial domain after some processing in the frequency domain, we must make sure to preserve both magnitude and phase of the Fourier image. Then, Retinex algorithm is applied followed by Bihistogram equalization and the output. image was obtained as the enhanced image. 


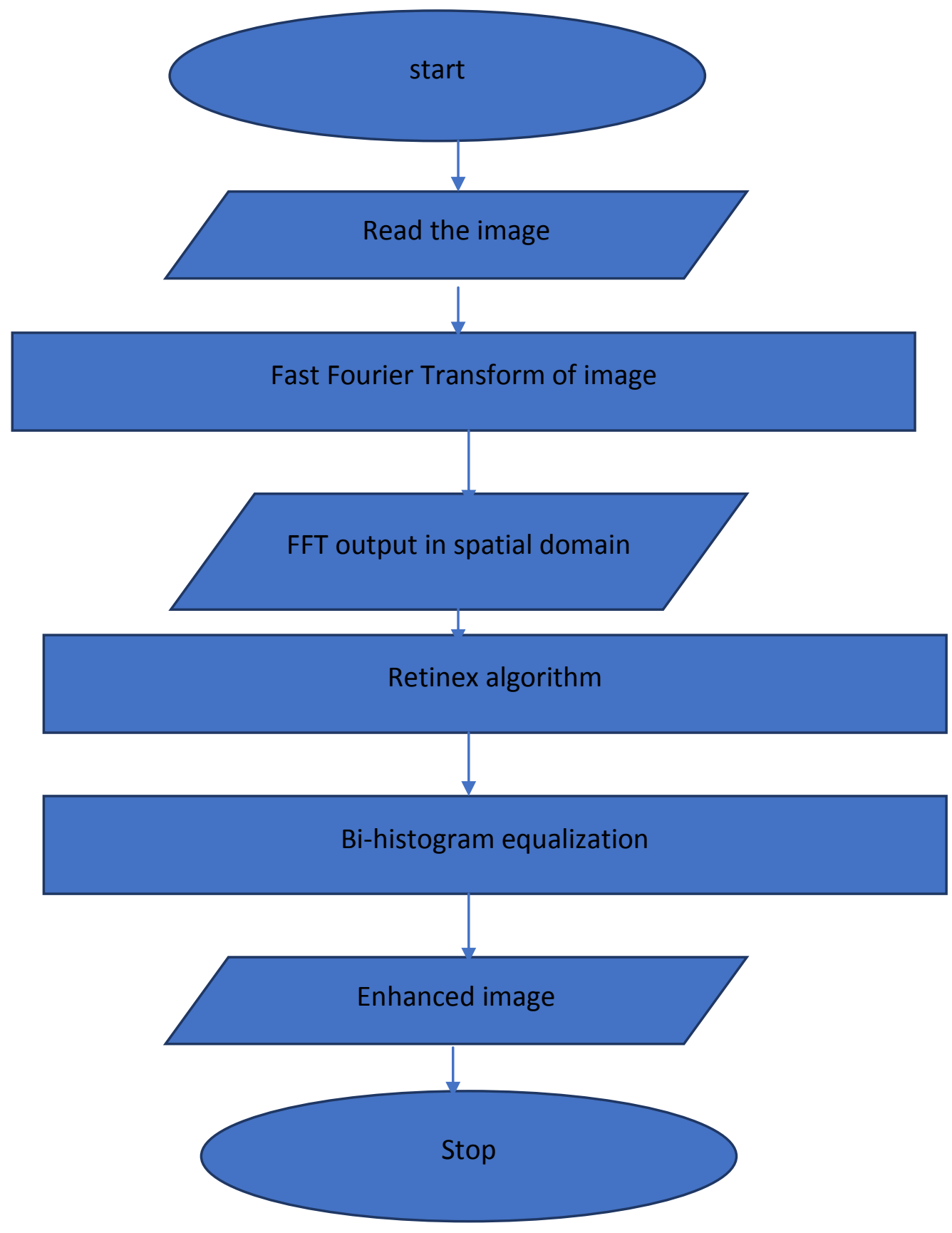

Figure 2 algorithm of the proposed system

$\mathrm{n}$ the figure 2, there are four main steps:

1. Read the color image (RGB) and extract the RGB separately

2. Apply Fast Fourier transform to each RGB and multiply it with gaussian filter, this will provide a more quality image, since applying filter in frequency domain yield a better result than apply it directly.

3. Find the reflectance image(output) using Retinex algorithm by subtracting the illumination image from original image in logarithm domain.

4. Bi Histogram Equalization (BHE) is used to enhance the contrast of the resultant image so as to optimize its visual effect. BHE first finds average point in histogram of the image and then divides the histogram in to two segments based on the point. After that histogram equalization operation is applied on each segment.

\section{EXPERIMENTAL EVALUATION}

To validate the proposed system, we used MATLAB 2018b to test the method by reading the images with resolution of $220 \times 220 \times 3$ color image and hardware environment used: core i7 OS with $2.9 \mathrm{Ghz}$

\subsection{Subjective analysis}

The following figures are the testing results. There are original image, enhanced image by MSR and enhanced image by our proposed system. In figure 3, the original image has low illumination and is somehow blur, after the enhancement of the two algorithms, the result show that our system has better visual effect compared with MSR. 

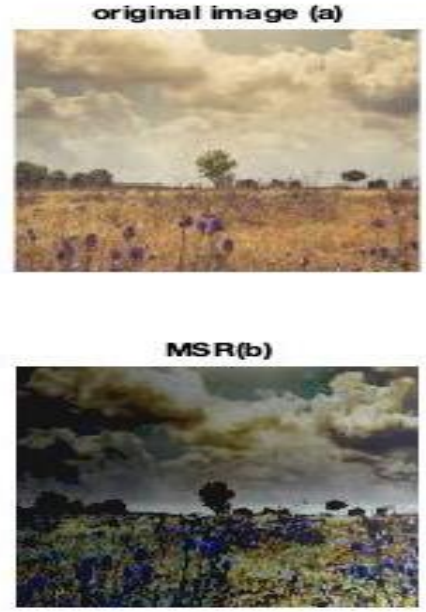

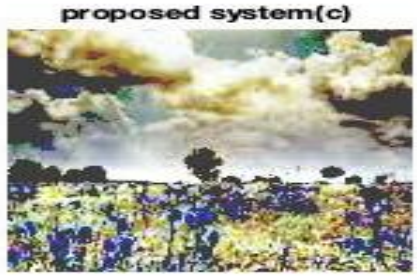

Figure 3

In figure 4 , the original image is blur, low illuminated and halation can be found. However, the two enhanced algorithms improved the illumination but halation was found in the MSR
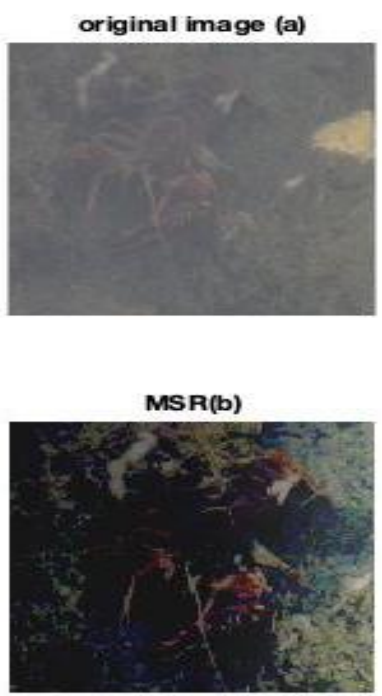

result, whereas halation has been eliminated in the propose system.

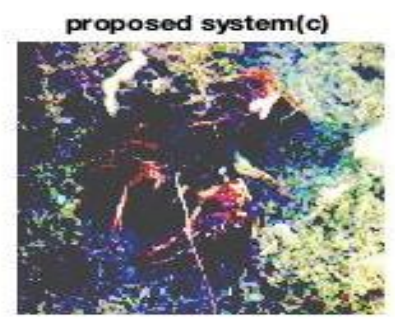

Figure 4

with more details. Mean square error shows the different between the enhanced image and original image, so small MSE means the enhanced image is similar with original image. The proposed system takes less computational time compared with MSE.

\subsection{Objective analysis}

To evaluate performance of objective analysis, we used entropy, mean square error (MSR) and time taken to determine how good the proposed system. Entropy indicates the scale of the information; the large value shows rich image

Table 1 Evaluation of objective analysis

\begin{tabular}{|c|c|c|c|}
\hline Figures & Parameters & MSR & Ours system \\
\hline \multirow{3}{*}{ Figure3 } & Entropy & 7.4848 & 7.4831 \\
\cline { 2 - 4 } & MSE & $4.6508 \mathrm{e}+03$ & $2.5653 \mathrm{e}+03$ \\
\cline { 2 - 4 } & Time (ms) & 275 & 73 \\
\hline \multirow{2}{*}{ Figure4 } & Entropy & 5.6179 & 3.7314 \\
\cline { 2 - 4 } & MSE & $4.3146 \mathrm{e}+03$ & 65 \\
\cline { 2 - 4 } & Time(ms) & 200 & 3.883 \\
\hline
\end{tabular}


According to the results in Table 1, the proposed algorithm outperforms other approach in term of entropy, MSE and time, only in figure3, the proposed system got less Entropy.

\section{CONCLUSION}

In this paper, we proposed a fast and efficient image enhancement algorithm based on fast Fourier transform with Bi-histogram equalization, instead of traditional application of gaussian filter to an image. We applied the filter in frequency domain and optimize the visual effect with bi-histogram equalization. The result shows that the proposed system provided better improvement compared with MSR.

\section{REFERENCES}

[1] Tarun D., A fast method of contrast enhancement using histogram equalization, International journal of electronics and communication engineering, volume 4, number 4 (2011), pp. 383-390.

[2] Yeong-taekgi M., Contrast enhancement using brightness preserving bi- histogram equalization, IEEE transactions on consumer electronics, vol. 43, no. 1, February 1997.

[3] Yu W., Qian C., Baomin Z., Image enhancement based on equal area dualistic sub-image histogram equalization method, IEEE transactions on consumer electronics, vol. 45, no. 1, February 1999.

[4] Jobson D. J., Rahman Z., and Woodell G. A., "A multiscale Retinex for bridging the gap between color images and the human observation of scenes," IEEE Trans. Image Process., vol. 6, no. 7, pp. 965-976, July 1997.

[5] LI Fu-wen, JIN Wei-qi, CHEN Wei-li. Global Color Image Enhancement Algorithm Based on Retinex Model. Transactions of Beijing Institute of Technology, 2010, 8(8), pp. 947-951.

[6] Hu W., Wang R., Fang S., Hu Q.," Retinex Algorithm for Image Enhancement Based on Bilateral Filtering, 2010", journal of engineering graphics,2, pp. 104-109.

[7] Jason L. M., Marwan Y. A. and Evan H. "Advanced Image processing with DirectX ${ }^{\circledR} 9$ Pixel shaders" From
ShaderX2 -Shader Programming Tips and Tricks with DirectX9,2003

[8] Di 1., Zhang Y. Wen P., Bai L., "A Retinex Algorithm for Image Enhancement Based on Recursive Bilateral Filtering, 2015", international conference on computational intelligent and security

[9] Pizer et. al," adaptive histogram equalization and its variation, 1987" computer vision, graphics and image processing, vol 39, pp 355-368

[10] Pinaso et. al "contrast limited adaptive histogram equalization image Processing to Improve the Detection of Simulated Speculations in Dense Mammograms, 1998" Journal of Digital Imaging, Vol 11, pp 193-200

[11] Edwin H. L. and John J. M.," lightness and Retinex theory, 1971 "journal of optical society of America, Polaroid Corporation, Cambridge, Massachusetts 02139

[12] Prabhu M., Rajarajan S. and Karthikeyan M. P. "Implementing Histogram Equalization and Retinex Algorithms for Image Contrast Enhancement, 2014", International Journal of Applied Engineering research volume 9, Number 20 (2014) pp. 7311-7318

[13] Doo Hyun Choi, Ick Hoon Jang, Mi Hye Kim, and Nam Chul Kim," color image enhancement using single-scale Retinex based on an improved image formation model, 2008”, European Signal Processing Conference.

[14] Petro A. B., Catalina S., Jean-Michel M., Multiscale Retinex, Image Processing On Line, 4 (2014), pp. 71-88. http://dx.doi.org/10.5201/ipol.2014.107

[15] Lokesh B. S. et. al" Retinex processing for automatic image enhancement using wavelet transformation ,2018" International Journal of Advance Research, Ideas and Innovations in Technology, volume 4, Issue 3, pp 1623 1626

[16] Shiping M. et. al," A Low-Light Sensor Image Enhancement Algorithm Based on HSI Color Model ,2018”, Sensors 2018, 18, 3583; doi:10.3390/s18103583 www.mdpi.com/journal/sensors 